\title{
Design and Implementation of Stair-Climbing Robot for Rescue Applications
}

\author{
Basil Hamed
}

\begin{abstract}
For disaster mitigation as well as for urban search and rescue missions, it is often necessary to place sensors or cameras into dangerous or inaccessible areas to get better situation awareness for the rescue personnel, before they enter a possibly dangerous area. Robots are predestined to this task, but the requirements for such mobile systems are demanding. They should be quick and agile and, at the same time, be able to deal with rough terrain and even to climb stairs. This paper presents the design and implementation of a feedback control system for an RF remote-controlled stairclimbing robot. The robot is controlled using PIC 16F877A.The paper presents a complete integrated control architecture and communication strategy for a system of reconfigurable robots that can climb stairs. Its mechanical design is suitable with back wheel to drive the robot over rubble, and large wheels in the front driven by dc motor for climbing stairs. The operator can monitor the robot operation by using video that are captured through a camera on the surface of the robot. The robot system is implemented by using MikroC and visual basic programs. Experimental trials showed that the implementation of the behavior control systems was successful.
\end{abstract}

Index Terms-Control, Robot, PIC 16F877A, Wireless Communication

\section{INTRODUCTION}

Robots are increasingly being integrated into working tasks to replace humans. They are currently used in many fields of applications including office, military tasks, hospital operations, industrial automation, security systems, dangerous environment and agriculture [1].Several types of mobile robots with different dimensions are designed [2-8] for various robotic applications. The robot has been designed for the purpose of aiding rescue workers. Common situations that employ the robot are urban disasters, hostage situations, and explosions. The benefits of rescue robots to these operations include reduced personnel requirements, reduced fatigue, and access to unreachable areas [9-11]. The robot is designed to go into slightly destroyed areas to find and help rescue people. The robot is even made to climb stairs and travel through fairly large amounts of rubbles. On the robot there will be a camera which is used to take video. The robot is built to discover areas which people cannot reach. This robot is able to cope with stairs, very rough terrain, and is able to move fast on flat ground. The robot is wirelessly connected to a transmitter/receiver circuit through RF remote control unit ensuring fast and reliable two-way communication

The robot body was prepared mechanically and electrical components were chosen to be suitable for the task of the robot. The robot is controlled using microcontroller PIC $16 \mathrm{~F} 877 \mathrm{~A}$ as the brain of control. The motion of the robot is controlled by controlling the direction of motors then the robot can move in all directions (forward, reverse, right and left).The robot speed is controlled by generating PWM from the PIC, in addition to that the arm (front) of the robot can move up and down by controlling the servo motor, and the camera can move right and left by controlling the stepper motor.

The Stair-climbing Robot will be controlled by three different ways:

- First, the robot is controlled by using serial joystick, the serial joystick contain pushbuttons and potentiometer. This Pushbuttons control movement of motors (DC motor and stepper motor) and potentiometers control PWM.

- Second, the robot is controlled by interfacing stairclimbing robot with PC; in this way serial port was used. This port consists of two wires to transfer data (one for each direction) and a number of signal wires.

- Finally, RF module is used to control the robot wireless. $433.92 \mathrm{MHz}$ RF Transmitter and Receiver is wireless data transmit and receive module with VHF/UHF super high frequency. It has strong anti-static protection and high reliability.

In addition to that USART (Universal Synchronous Asynchronous Receiver Transmitter) is used.

\section{HARDWARE RoBot DESIGN}

The robot has been assigned the task of creating a robot capable of discovering the existence of humans or things that trapped inside of an unstable, collapsed buildings or not reachable area by human. This section will present a full description of the hardware of the robot design and is divided into two main sections: Mechanical and Electrical design.

\section{A. Mechanical Design}

The robot has dimension $(60 \times 40 \times 13) \mathrm{cm}$ which is the length, width, and height respectively, as shown in Figure (1).

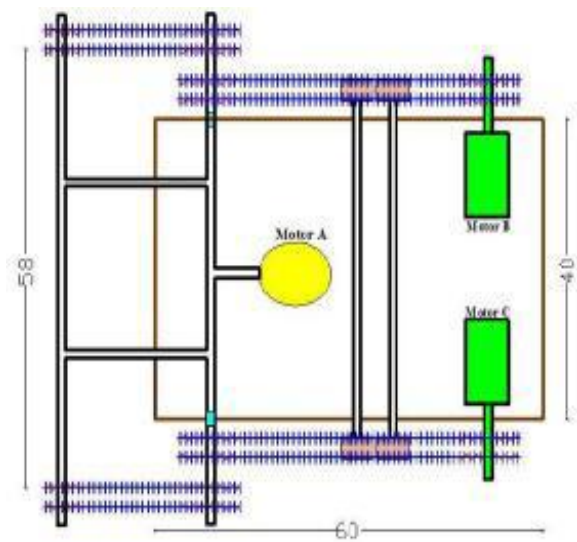




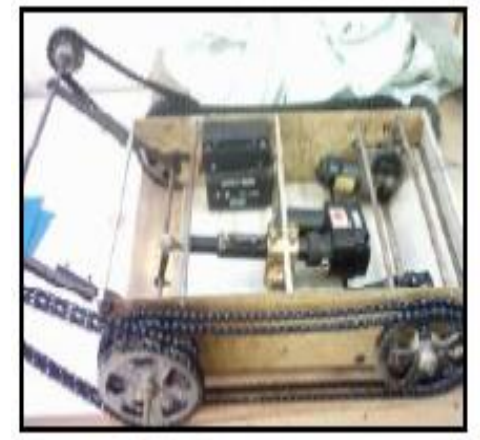

Figure (1) Top View of the Robot

\section{Gears and its Dimension}

The robot requires twelve large gears with diameter of 17 $\mathrm{cm}$, as shown in Figure (2) eight of them joined together for robot movement to enable the robot to travel through fairly large amounts of rubble,. The other four large gears join with four small gears with a diameter of $8 \mathrm{~cm}$ to be able to climb the stairs. We use a double bicycle chain to join the gears.

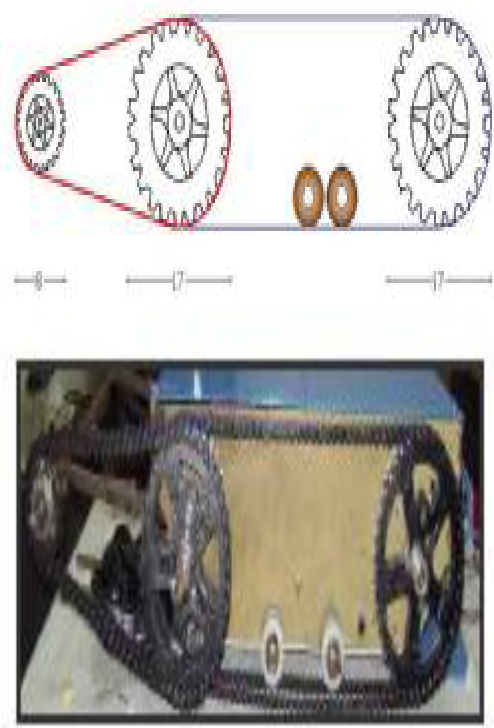

Figure (2) Gears of the Robot

\section{Robot Movement Mechanism:}

The robot mechanism drive works with the rear wheels being directly powered and the front wheel system being driven by the same motor via a series chain. The movement of the robot is established by using two motors in each side. A servomotor with arm (Satellite Dish Motor) is used to the case of climbing stairs by controlling the position of the motor arm which is joined with the front of the robot

\section{Advantages of Rear Wheel:}

- Maintenance is cheap and easy.

- Fixed in long lines and roads.

- It's Strong and less carrying havoc.

- More comfortable and stable.

- Non-pressure build-up on the front wheels.

- Rear wheel has the task of spin, receiving torque and acceleration, which leads to better control of the robot.

- The front wheel is responsible for the direction and the rear back wheel is for push

\section{Extra Mechanical Parts}

Iron and wood are used for the body of the robot because they are strong and can bear the motor weight and movement. The outside cover of the robot is fiber plastic because it is light. Plastic wheels are used to make chain always in a down the body even when climbs stairs. The robot is supported by a wireless camera at the top of its surface connected by a stepper motor to control its movement as shown in Figure (3).

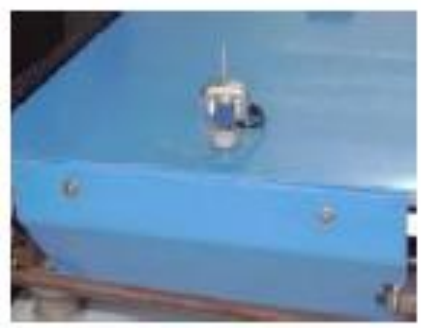

Figure (3) Camera Attached To Stepper Motor

\section{B. Electrical Design}

\section{Block Diagram}

The block diagram shown in Figure (4) presents the main structure of the stair-climbing robot which consists of: Power sources, charger circuit, motors (DC motor, Servo motor, Stepper motor), and wireless and wire modules. The brain of the robot is PIC16F877A. The following sections describe in details each of these components.

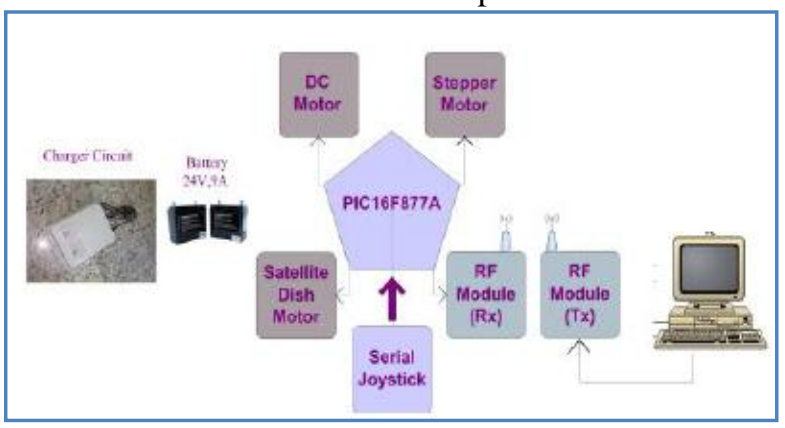

Figure (4) Block Diagram of the Stair-Climbing Robot

\section{Power Sources}

Primary source of power for the robot are lithium batteries (Li-ion) because of its characteristics and advantages. Two batteries (12 Volt 9 Ampere) deliverers, respectively, to provide 24 volts and Can recharge this batteries using charger circuit.

\section{Power Circuit}

Circuit shown in Figure (5) is used to supply the robot with different values of voltages from batteries:

- Directly from batteries 24 volt for DC motors and Satellite Dish motors.

- Using (LM7812) it generates 12 volt.

- Using (LM7808) it generates 8 volt for wireless camera.

- Using (LM7805) it generates 5 volt for control circuit.

\section{Charger Circuit}

The circuitry to recharge the batteries in a portable product is an important part of any power supply design. The complexity (and cost) of the charging system is primarily dependent on the type of battery and the recharge 
time.

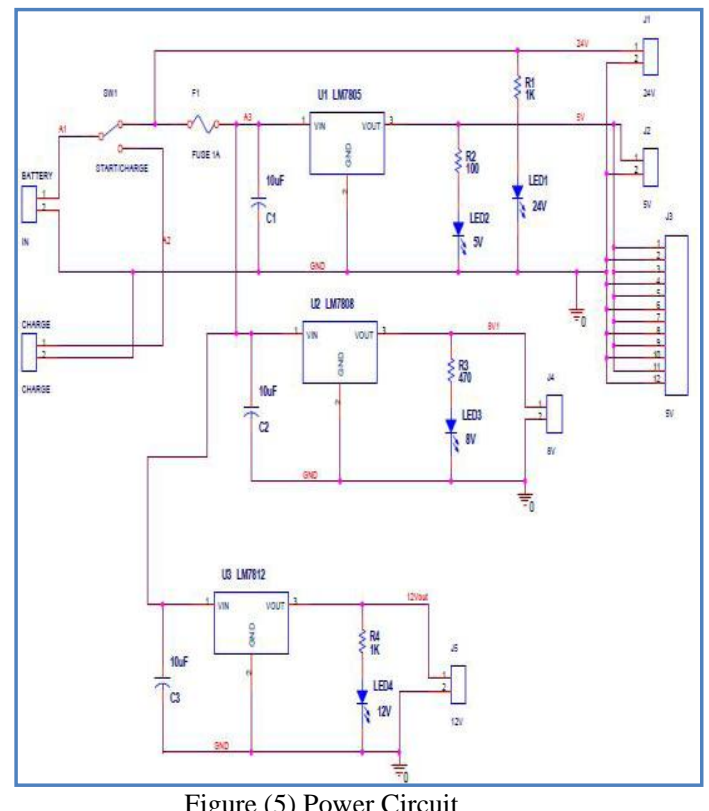

As shown in Figure (6-b)when TR1 is set correctly then the next round of charging it will be notice D1 begin to flicker as the battery is being charged. When battery is completely charged, D1 turns ON completely.TR1 do not need further adjustment anymore. Q1 is connected in line with the battery and is fired by R2, R3and D1. The R4, C1, TR1 and D3 sense the voltage of the battery terminal and activate Q2 when the voltage of the battery terminal exceeds the value predetermined by TR1. When an uncharged battery is connected, the terminal voltage is low. Under this circumstance, Q2 is turned OFF and Q1 is fired in each half cycle by R2, R3 andD1. The Q1 functions as a simple rectifier and charges the battery. When the battery terminal voltage is increased the level that had been fixed by TR1will makes Q2shifts the control of Q1 gate. This deactivates Q1 and cuts off the current supply to the battery and turns D1 ON indicating that the charge has been completed. M1 is a $3 \mathrm{ADC}$ ammeter to measure the charge current. The AC/DC controlled rectifier provides a variable DC load voltage from a fixed voltage and frequency source $(220 \mathrm{~V} \mathrm{ac}, 50 \mathrm{~Hz})$ as shown in Figure (6-a).

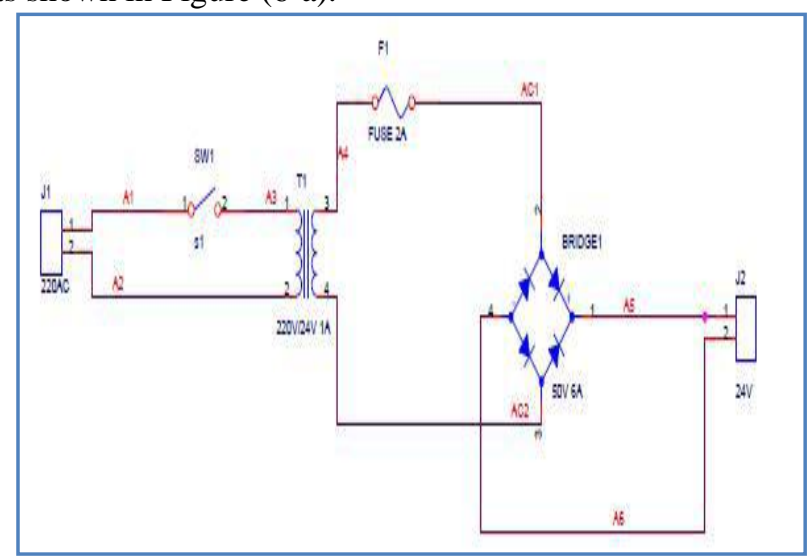

Figure (6-a) Full wave Rectifier (AC/DC Converter)

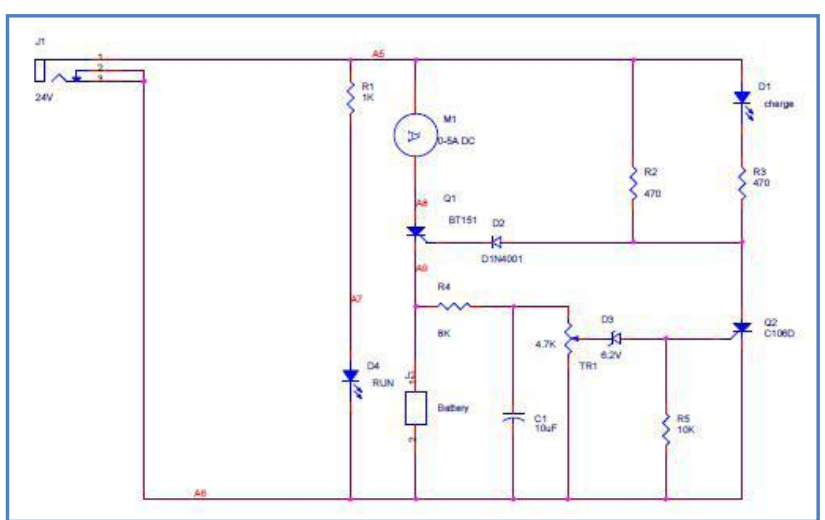

Figure (6-b) Charger Circuit

\section{SOFTWARE RoBot DESIGN}

The robot software complements the hardware architecture of the stair-climbing robot by providing basic low-level hardware control that include reading the sensors value and controlling the motor speed.

Mikro C Program: Mikro C is a powerful, feature rich development tool for PIC microcontroller. It is designed to provide the programmer with the easiest possible solution for developing applications for embedded systems, without compromising performance or control. [12][13].

Proteus Program: Provides detailed instructions on how to create new simulator models, using both schematic and programmatic (DLL) based techniques. It is an interactive system level simulator (Figure 19). Which combines mixed mode circuit simulation, micro-processor models and interactive component models to allow the simulation of complete micro-controller based designs. [14]

OrCAD PCB Program: used for PCB design. An interactive environment for creating and editing simple to complex multi-layer PCBs, it uses powerful shape-based algorithms for speed and efficient use of the routing area. OrCAD facilitates rapid design-and-simulate cycles, allowing engineers to explore various design configurations before committing to a specific circuit implementation [15].

Visual Basic Program: used for Interfacing between PC and the stair-climbing Robot. Microsoft Visual Basic 6.0 is used in this paper because of its easy programming, easy displaying of visual elements, availability. it is one of the most popular programming languages and it is easy to implement functions using it [16].

\section{PIC16f877A}

PIC16f877A is used as the brain of the robot that can be programmed by connecting the serial port of the computer to the PIC microcontroller. The serial port operates at $+/$ $13 \mathrm{~V}$, and the PIC serial operates at $+5 \mathrm{~V} / 0 \mathrm{~V}$ [17]. MAX232 is used as a level shifter to connect the serial port of the computer to pins RX/TX on PIC as shown in Figure (7). 


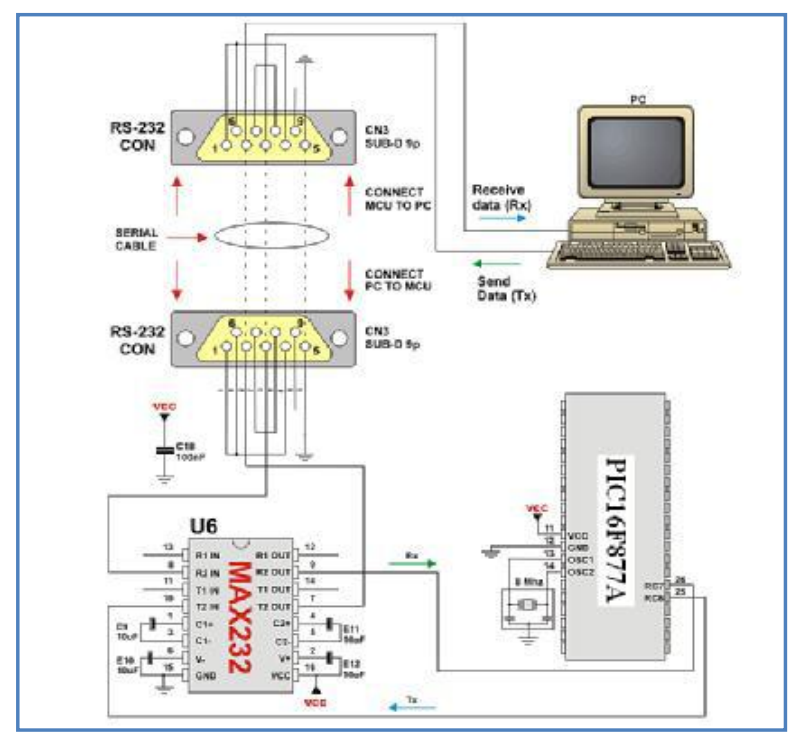

Figure (7) PIC Interface with Serial Port

\section{Motor control}

DC Motor shown in Figure (8) is used as the power engine of the stair-climbing robot. The stair-climbing robot needs high torque with minimum current for the robot to be able to climb stairs. Two types of motors are needed: wiper motors and brushless DC motors. That is, minimum current and high speed is supplied from brushless DC motors, and high torque is supplied from the gears of wiper motors.

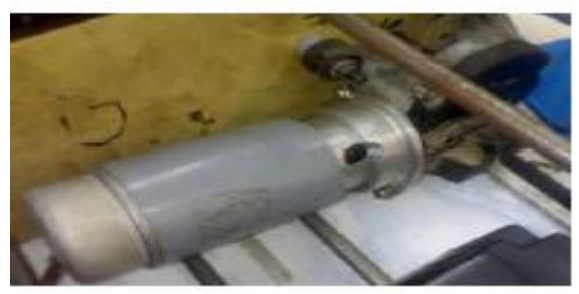

Figure (8) DC Motor

\section{Direction and Speed Control}

H-bridge as shown in Figure (9) is used to control a DC motor speed and direction. It allows the motor to start and stop and most importantly to reverse direction. In order to reverse the direction of a DC motor, the current through the DC motor must be reversed.

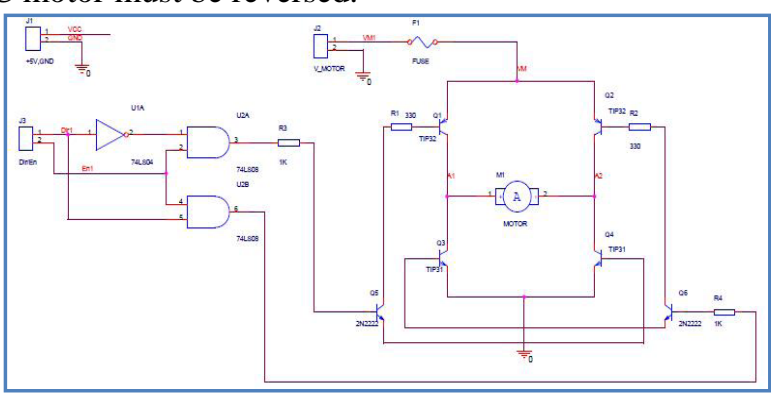

Figure (9) H-Bridge Implementation

\section{H-Bridge Operation}

- $\quad$ TIP42 (PNP) will be operated when VBE $<0.7$.

- $\quad$ TIP41 (NPN) will be operated when VBE $\geq 0.7$.

- Q5 will be operated when the output of (U2A 74L808) is set.

- Q6 will be operated when the output of (U2B $74 \mathrm{~L} 808$ ) is set.
- When Q5 is operated Q1 and Q4 will be operated (rotates left).

- When Q6 is operated Q2 and Q3 will be operated (rotates right).

\begin{tabular}{|l|l|l|l|l|l|l|}
\hline \multicolumn{1}{|c}{ TABLE (1) OPERATION OF H-BRIDGE } \\
\hline 0 & 0 & 0 & 0 & 0 & 0 & Stop \\
\hline 0 & 1 & 1 & 0 & 0 & 1 & Qeft \\
\hline 1 & 0 & 0 & 0 & 0 & 1 & Stotor \\
\hline 1 & 1 & 0 & 1 & 1 & 0 & Right \\
\hline
\end{tabular}

\section{PWM Generation}

To control the speed of a D.C. motor a variable voltage D.C. power source is needed. When a $12 \mathrm{~V}$ motor switches on, the motor will start to speed up. Motors do not respond immediately so it will take a small time to reach full speed. When power switch off sometime before the motor reaches full speed, the motor will start to slow down. When switch the power on and off quickly enough, the motor will run at some speed part way between zero and full speed. This is exactly what a PWM controller does: it switches the motor on in a series of pulses. To control the motor speed it varies (modulates) the width of the Pulse Width Modulation.

Code

Purn Init (250):

Pwr_Change_Duty (127):

Porm Start $(\overline{)}$ :

\section{Description}

- Pwm_Inti (250) Pwm_Inti (unsigned long frequency) the value of frequency dependent on the value of frequency of crystal.

- Pwm_Change_Duty(127) Pwm_Change_Duty (unsigned short duty_ratio)

- Parameter duty takes values from 0 to 255 , where 0 is $0 \%, 127$ is $50 \%$, and

- 255 is $100 \%$ duty ratio. Other specific values for duty ratio can be calculated as (Percent*255)/100.

- Pwm_Start() Starts PWM .

\section{Servo Motor Control:}

Servo motor is one of the DC type motors with feedback that used in many applications that required controlling the system in up-down direction. Servos are extremely useful in robotics [18]. In order to let the robot climb and go down the stairs (up-down direction), servo motor is used. In the servo motor position control system there is two set points and two sensors (limit switches) used to feedback the position information to the controller. 


\section{L298N Dual Full-Bridge Motor Driver}

L298 is a dual H-bridge driver as shown in Figure (10) for DC brushed motors and stepper motors. It supports a wide operating voltage range and can deliver 2 A per channel in a through-hole package that is accessible for doit-yourself projects.

\section{L298N Features and Specifications}

- Operation to $46 \mathrm{~V}$

- Up to 2 A per channel

- Outputs can be paralleled to drive up to $3 \mathrm{~A}$

- Independent ground connections for each channel allow independent current sensing.

- Multiwatt15 through-hole package allows convenient heat sink mounting and easy prototyping with 0.1 " breadboards.

- L298N can control 2 DC Motors, their direction using control lines and there Speed using PWM.

- Dish Satellite motor has a constant speed that is why it has only direction control.

- The outputs of the two motors are connected in parallel to drive only one motor to maximize the output current of the bridge (3A).

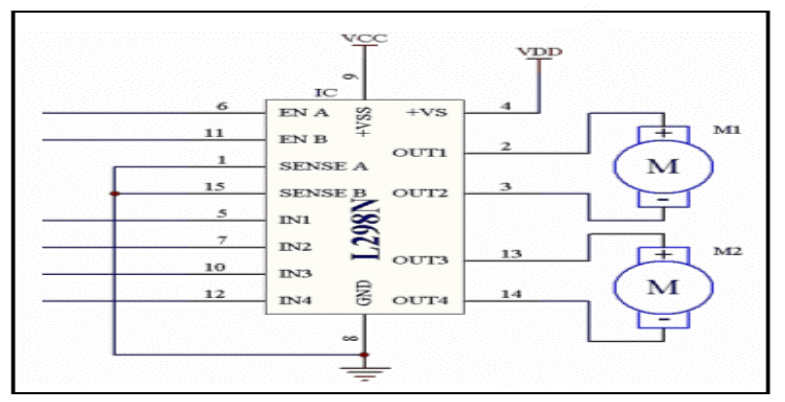

Figure (10) L298 Dual Full-Bridge Driver

\section{Stepper Motor Control}

A stepper Motor is an electromechanical device which converts electrical pulses into discrete mechanical movement. The shaft or spindle of a stepper motor rotates indiscrete step increment when electrical command pulses are applied to it in the proper sequence. The sequence of the applied pulses is directly related to the direction of the motor shaft rotation [19]. The main advantage of stepper motors is that they can achieve accurate position control without the requirement for position feedback. Stepper motor is used for the rotation of the camera in both Left and Right directions.

\section{Uln2003 - Seven Darlington Arrays}

ULN2003 is High Voltage / High Current Darlington Transistor Arrays; it's monolithic high voltage and high current Darlington transistor arrays. It consists of seven NPN Darlington pairs that feature high-voltage outputs (50V) with common cathode clamp diode for switching inductive loads. The collector-current rating of single Darlington pair is $500 \mathrm{~mA}$. The Darlington pairs may be paralleled for higher current capability. Applications include relay drivers, hammer drivers, lamp drivers, display drivers (LED gas discharge), line drivers, and logic buffers [20]. Its inputs pinned opposite outputs to simplify layout. This is more adequate to control a four phase unipolar stepper motor.

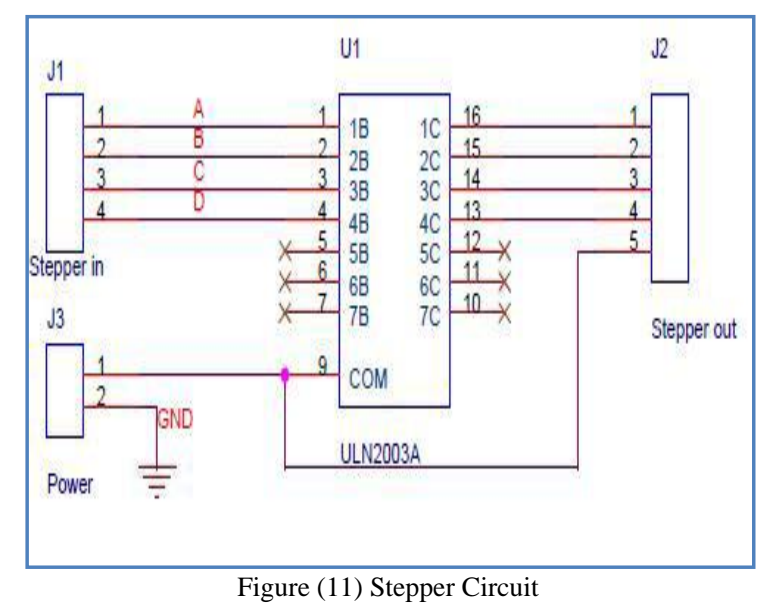

Code

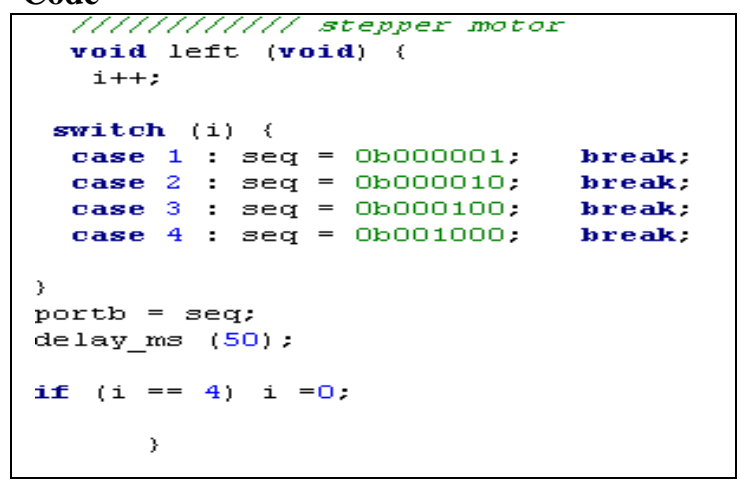

\section{Description}

$\mathrm{i}=0$ is an initial value, which will be incremented gradually from the statement $\mathrm{i}++$; this causes the sequence to be $\{$ case 1 , case 2 , case 3 , case 4$\}$ and the motor to rotate Left.

\section{Serial Joystick}

The movement of the stick of the joystick presents varying of the value of the potentiometer in either sides $\mathrm{X}$ or $\mathrm{Y}$, As shown Figure (12) where the maximum value in the most forward or most right is $4.8 \mathrm{~V}$, and the minimum value is $0 \mathrm{~V}$,otherwise at its home position it has a value of $2.5 \mathrm{~V}$. On the other hand the pushbuttons give on/off voltage values 0 or $5 \mathrm{~V}$. These Push buttons control the movement of motors (servo motor and stepper motor) and potentiometers control the speed of DC motors.

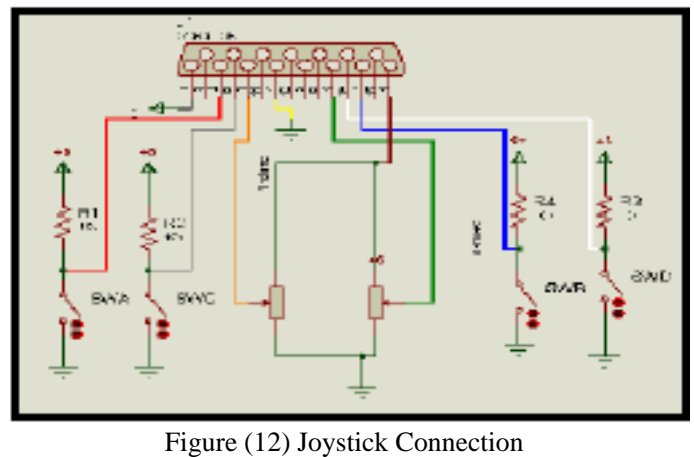

\section{Interface between stair-climbing robot and $\mathrm{PC}$}

Personal computers (PC) have a large number of ports that you could add your own hardware to control stairclimbing robot. Some of these are very easy to use, while others are nearly impossible without special (expensive) ICs. Not all of these interfaces are available on all computers. 


\section{Serial Port}

The serial port as shown in Figure (13) is one of the two easiest to use ports on a PC. This port consists of 2 wires to transfer data (one for each direction) and a number of signal wires. This port is reasonably sturdy, and knowing digital electronics and how to use a microcontroller, is pretty easy to use. It is limited on speed and can only connect two devices directly.

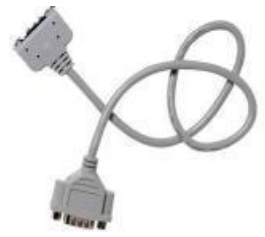

Figure (13) RS-232 Serial Communication

\section{USART}

USART stands for Universal Synchronous Asynchronous Receiver Transmitter. It is sometimes called the Serial Communications Interface or SCI. Synchronous operation uses a clock and data line while there is no separate clock accompanying the data for Asynchronous transmission. Since there is no clock signal in asynchronous operation, one pin can be used for transmission and another pin can be used for reception. Both transmission and reception can occur at the same time - this is known as full duplex operation. Transmission and reception can be independently enabled. However, when the serial port is enabled, the USART will control both pins and one cannot be used for general-purpose $\mathrm{I} / \mathrm{O}$ when the other is being used for transmission or reception. The most common use of the USART in asynchronous mode is to communicate to a PC serial port using the RS-232 protocol. A driver is required to interface to RS-232 voltage levels and the PIC; MCU should not be directly connected to RS-232 signals [21]. MAX232 is used as a driver (voltage level shifter) as explained earlier.

\section{USART Library:}

USART hardware module is available with PIC16F877A .This library is used to communicate between PC and PIC16F877A, thus we enabled to control the robot via mouse or USB joystick

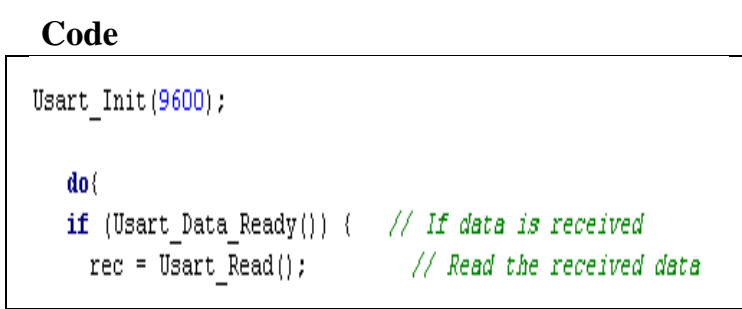

\section{Description}

- USART_InitInitializeshardware USART module with the desired baud rate.

- UsartData_Ready () USART_ReadIf data is ready, read it.

Then the received data will be examined and the proper function will be executed.

\section{Visual basic Programming}

The design of any successful robot involves interface software with PC. Visual Basic program is one of the best to implement this function. Connection to the RS-232 port is accomplished with the Visual Basic standard MSCOMM control. By using these features of Visual Basic the code is completely transportable. Through a series of buttons and commands on the interface software we can control all movements of the robot as shown in Figure (14). It consists of frame Movement of robot control direction of two DC motors forward or reverse, left or right, as well as control servomotor. Frame Direction of Camera to control Stepper motor that move camera left or right and frame audio video control (A/V Control) used to control the function of camera as shown in Figure (15).

\section{Code}

Private Sub Form Load! !

MSComm1. RThreshold $=2$

IISComm1. InputLen $=2$

' 9600 Baud, No Parity, 8 Data Bits, 1 Stop Bit

MSComm1. Settings $=" 9600, \mathrm{~N}, 8,1 " \quad 1$ Disable DTR

ISCorm1. DTREnable $=$ False 1 Open CoM1

MSCorm1. Cormport $=1$

ISTCorm1. Portopen $=$ True

End Sub

Private Sub Command1_Click()

ISSCom1. Portopen $=$ Fälse

Unload Form1

End Sub

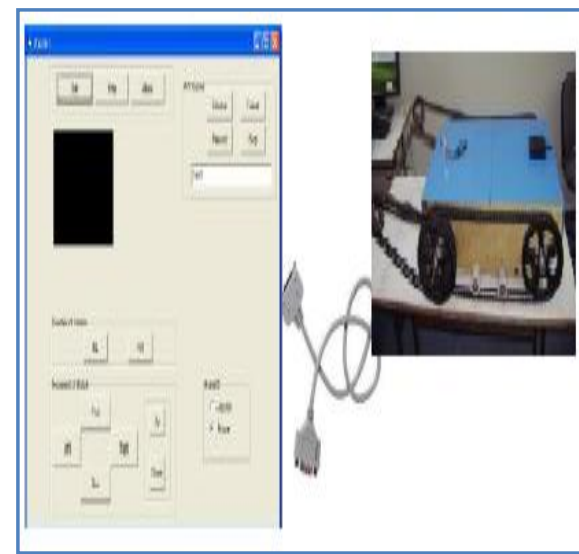

Figure (14) Serial Connection between PC and Stair-Climbing Robot

\section{Description}

- Initializing and Opening the Com port, Create an instance of CRs232 then set COM parameters before invoking the Open method.

- Transmitting data to COM Port: when continue pressing on the command sends serially signal.

- Joystick.ocx to read from USB joystick.

- Resize.ocx to zoom out interface software.

- ezVidCap.ocx to control functions of camera.

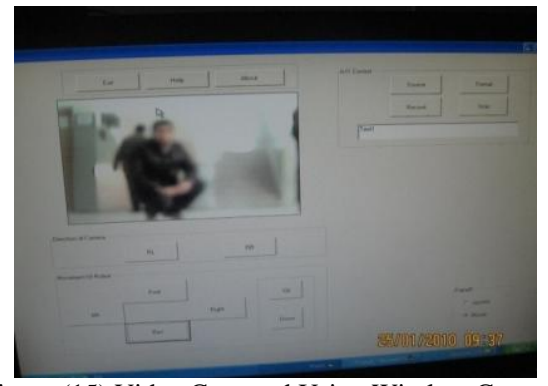

Figure (15) Video Captured Using Wireless Camera 


\section{WIRELESS COMMUNICATION}

Wireless communication involves the electromagnetic spectrum, which has many unique qualities. Radio waves propagate according to the spectrum's wavelength. Antennas provide an impedance match to the airwaves. The RF Module provides a convenient interface for wireless it's allow engineers of all skill levels to quickly and costeffectively add wireless capabilities to virtually any product. It consists of two IC's TX and RX. It can be installed in a suitable location up to 100 meters cabling distance from the Control Module. This allows a greater degree of flexibility for optimizing the location of the RF receiver for the best coverage. The robot remote control in this project in one direction to send a signal from the PC and received by the PIC on the body of the robot as shown in Figure (16).

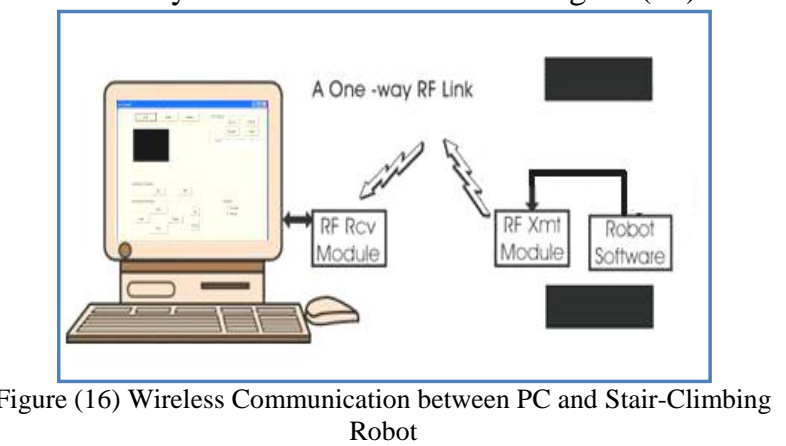

\section{Connection of RF Module in Stair-Climbing Robot}

In Stair-Climbing Robot TX is located near the computer connected to it by a cable from the computer to max circuit where the TX locate, RX is located on the surface of the Stair-Climbing robot as shown in Figure (17), in order to control the movement of the robot from the wave transmit from the computer throw TX to the robot throw RX.

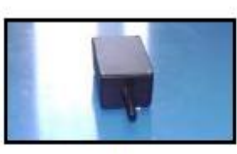

(a)

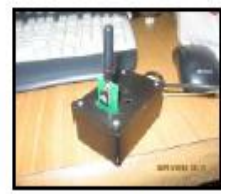

(b)
Figure (17) Connection of RF Module in Stair-Climbing Robot

As explained previously the stair-climbing robot can be controlled wired through the joystick or via a serial port between the PC and PIC as shown in Figure (7) or wireless via $\mathrm{RF}$ module that connect directly with PIC without MAX232 as shown in Figure (18). This adjustment manually on the PIC circuit by connectors

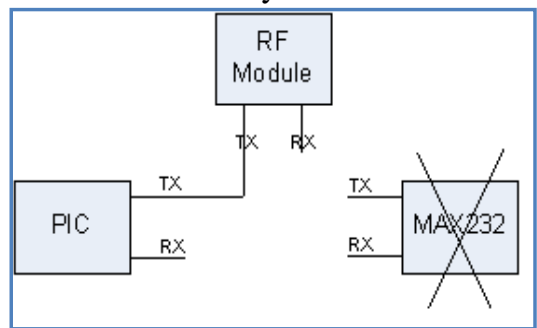

Figure (18) Wireless Connection

\section{CONCLUSIONS}

The robot is called stair-climbing robot from the fact that it's designed to cope with stairs, very rough terrain, and is able to move fast on flat ground. To sum up, the main concern of this paper is to design a rescue robot that is capable to go into slightly destroyed areas to find and help rescue people.PIC16F877 is used in this robot in order to control the direction (right, left, forward and reverse) using two DC motors in both sides of the robot using H-bridge as driver. Also PIC is used to control the motion of camera using stepper motor and the motion of servo motor which is used to let the robot climb and go down the stairs. PIC is the brain of stair-climbing robot. The overall system worked successfully. Firstly we tested our robot using serial joystick (wired system).Then RF module (transmitter and receiver) is used in order to make the system wireless by using USB joystick control. The control used was by making interface between PIC and visual basic in both directions; sending data from computer to robot or vice versa. Overall benefits of rescue robots to these operations include reduced personnel requirements, reduced fatigue, and access to unreachable areas

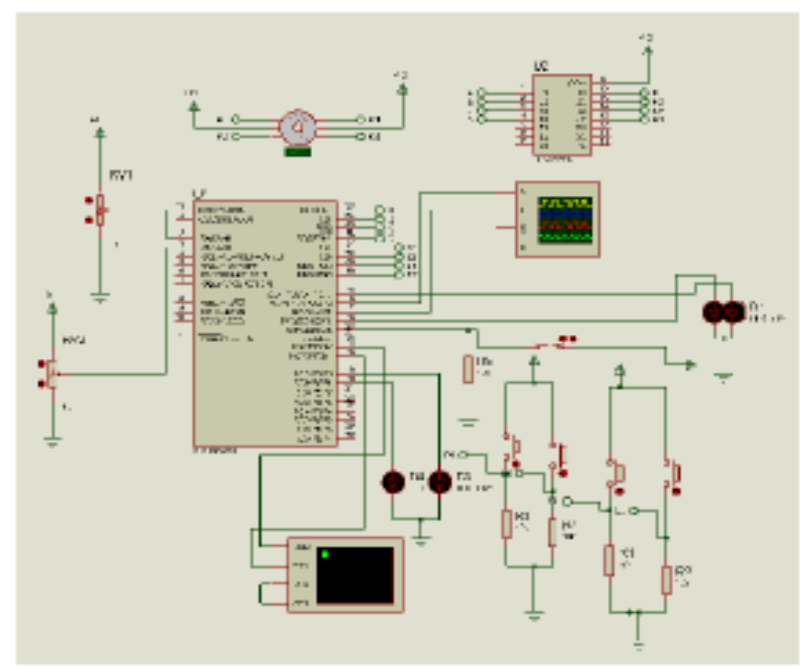

Figure (19) Stair-Climbing Robot Proteus Simulation
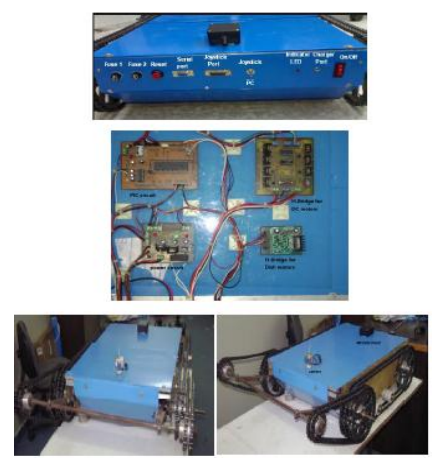

Figure (20) Stair-Climbing Robot

\section{REFERENCES}

[1] R. C. Luo , K. L. Su, "Amultiagentmulti sensor based real-time sensory control system for intelligent security robot" IEEE International Conference on Robotics and Automation, vol. 2, 2003, pp.2394-2399.

[2] G. T. Sibley, M. H. Rahimi, G. S. Sukhatme, "Robomote: a tiny mobile robot platform for large-scale ad-hoc sensor networks", IEEE International Conference on Robotics and Automation, ICRA 'O2, vol.2,2002, pp.1143-1148.

[3] S. Bergbreiter, K. S. J. Pister, "Cots Bots: an off-the-shelf platform for distributed robotics", IEEE/RSJ International Conference on Intelligent Robots and Systems, IROS '03, vol.2, 2003, pp.1632-1637. 
[4] A. Arora, E. Ertin, R. Ramnath, M. Nesterenko, W. Leal, "Kansei: high-fidelity sensing tested", IEEE Internet Computing, vol.10, 2006,pp. 35- 47.

[5] H. Utz, S. Sablatnog, S. Enderle, G. Kraetzschmar, "Miromiddlewarefor mobile robot applications", IEEE Transactions on Robotics and Automation, vol.18, 2002, pp. 493- 497.

[6] G. Caprari, K. O. Arras, R. Siegwart, "The autonomous miniature robot Alice: from prototypes to applications", IEEE/RSJ International Conference on Intelligent Robots and Systems, IROS 2000, vol.1, 2000, pp. 793-798.

[7] G. Metta, P. Fitzpatrick, L. Natale,YARP: "Yet another Robot Platform”, International Journal of Advanced Robotic Systems, vol. 3, 2006, pp.43-48.

[8] Kalantari, A. Mihankhah, E. Moosavian, S.A.A. "Safe autonomous stair climbing for a tracked mobile robot using a kinematics based controller" AIM. IEEE/ASME International Conference on Advanced Intelligent Mechatronics, 2009.

[9] Akhtaruzzaman, M.; IzzatiBt Samsuddin, N.; Bt Umar, N.; Rahman, M.;" Design and development of a wall climbing Robot and its control system" 12th International Conference on Computers and Information Technology, 2009. ICCIT '09.

[10] Sung Kyun Lim Dong Il Park Yoon Keun Kwak Byung-Soo Kim Sang-Won Jeon, “ Variable geometry single-tracked mechanism for a rescue robot” , Workshop, 2005 IEEE International Safety, Security and Rescue Robotics.

[11] Gaston, J. Raahemifar, K. Hiscocks, P “ A cooperative network of reconfigurable stair-climbing robots”, ISCAS 2006. Proceedings. IEEE International Symposium on Circuits and Systems, 2006.

[12] PIC Microcontrollers 1st EDITION (2008) Milan Verle, mikroElektronika

[13] Muhammad Ali Mazidi, Rolin D. Mackinaly, Danny Causey, "PIC MICROCONTROLLER AND EMBEDDED SYSTEMS Using Assembly and C for PIC18," New Jersey, Prentice Hall, 2006.
[14] Proteus Professional PCB Design and Simulation www.labcenter.co.uk/

[15] http://www.cadence.com/products/orcad/orcad_pcb_designer/pages/d efault.aspx

[16] Osama El Huseni, "Visual Basic for Windows," IbnSina Library, 1994.

[17] PIC16F87XA Datasheet, Pin Enhanced Flash Microcontrollers, Microchip Technology Inc., 2003.

[18] Riazollah Firoozian," Servo Motors and Industrial Control Theory" Springer; 1st edition (December 8, 2008)

[19] Kenjo, Takashi,"Stepping motors and their microprocessor controls" Oxford University Press, c1984.

[20] http://www.datasheetdir.com/ULN2003+Darlin gton-TransistorArrays

[21] http://www.alldatasheet.com/datasheetpdf/pdf25575/STMICROELE CTRONICS/ULN2003.html

Dr. Basil Hamed is Assistant Professor of Electrical Engineering Department, Islamic University of Gaza, Palestine, since 1999. He has Bachelor Degree in Electrical Engineering from New Mexico State University, NM. USA in the year of 1989, he received Master degree from University of New Orleans, La. USA in the year of 1992, and earned his PhD from New Mexico State University, NM USA in the year 1999. He has 15 years of teaching experience and has published many papers in national and international journals. His fields of interest include Control Systems, Fuzzy Control, Simulation \& Modeling, Robotics, FPGA, Signal and Image Processing. 